\title{
Research Article \\ Efficacy of Adaptive Biofeedback Training in Treating Constipation-Related Symptoms
}

\author{
Jing Tang, ${ }^{1}$ Zhihui Huang, ${ }^{2,3}$ Yan Tan, ${ }^{1}$ Nina Zhang, ${ }^{2,4}$ Anping Tan, \\ Jun Chen, ${ }^{1}$ and Jianfeng Chen ${ }^{5}$ \\ ${ }^{1}$ Division of Gastroenterology, Affiliated Hospital of Hainan Medical College, Haikou 571000, China \\ ${ }^{2}$ Ningbo Pace Translational Medical Research Center, Beilun, Ningbo 315000, China \\ ${ }^{3}$ Department of Gastroenterology, Sir Run Run Shaw Hospital, School of Medicine, Zhejiang University, Hangzhou 310000, China \\ ${ }^{4}$ Divison of Gastroenterology, The First Affiliated Hospital of Nanjing Medical University, Nanjing 210000, China \\ ${ }^{5}$ Ningbo Medkinetic Inc., Ningbo 315000, China
}

Correspondence should be addressed to Jing Tang; 13006003523@163.com and Zhihui Huang; huangzhihui808@gmail.com

Received 18 July 2014; Accepted 26 August 2014

Academic Editor: Jiande Chen

Copyright (C) 2015 Jing Tang et al. This is an open access article distributed under the Creative Commons Attribution License, which permits unrestricted use, distribution, and reproduction in any medium, provided the original work is properly cited.

\begin{abstract}
Biofeedback therapy is a well-known and effective therapeutic treatment for constipation. A previous study suggested that adaptive biofeedback $(\mathrm{ABF})$ training was more effective than traditional (fixed training parameters) biofeedback training. The aim of this study was to verify the effectiveness of $\mathrm{ABF}$ in relieving constipation-related symptoms. We noticed that in traditional biofeedback training, a patient usually receives the training twice per week. The long training sessions usually led to poor compliance. This study proposes an intensive biofeedback therapy and compares intensive therapy with nonintensive therapy in patients with constipationrelated symptoms. Methods. 63 patients with constipation-related symptoms were treated with ABF between 2012 and 2013 . These patients were further divided into the intensive therapy and nonintensive therapy groups. Results. A total of 63 patients were enrolled in the study, including 24 in the nonintensive therapy group and 39 in the intensive therapy group. $100 \%(N=21)$ of constipation patients achieved the primary efficacy endpoint ( $\geq 3$ bowel movements/week). There was significant improvement in constipationrelated symptoms after adaptive biofeedback. The intensive biofeedback therapy did not show better performance compared to nonintensive biofeedback therapy. Conclusions. This investigation provides support for the efficacy of biofeedback for constipationrelated symptoms. The efficacy of intensive therapy is similar to nonintensive therapy.
\end{abstract}

\section{Introduction}

Chronic constipation is a common disorder characterized by defecation difficulty or decreased bowel movements (less than three times a week). The worldwide prevalence of chronic constipation varies from $12 \%$ to $17 \%$ [1]. It is more prevalent in females than males (prevalence rate of $2.2: 1$ ) and the prevalence increases with age [2]. Patients who reported persistent constipation have decreased health-related quality of life and higher level of depression [3]. Chronic constipation has a great economic and social impact, including laboratory tests, diagnostic procedures, and healthcare expenditures [4].

Constipation is primarily a functional disorder, and it could also be caused by medications, diseases of the colon, metabolic disturbances, and neurologic disorders. Constipation can be categorized into 3 subgroups (obstructed defecation, slow transit constipation, and normal transit constipation) $[5,6]$. About $40 \%$ of constipation is due to obstructed defecation $[7,8]$. Obstructed defecation (also known as dyssynergic defecation, pelvic floor dyssynergia, or outlet obstruction) is characterized by the lack of coordination between the abdominal and pelvic floor muscles during defecation. Obstructed defecation is caused by one of the following problems: impaired rectal contraction, paradoxical anal contraction, or inadequate anal relaxation.

Although currently available treatment options have been reported to be effective at improving patients' symptoms, the curative effect is still unsatisfactory. There is insufficient data 
to support that lifestyle and diet change such as increased fiber and fluid intake can improve chronic constipation. Laxatives (including bulking agents, osmotic and stimulant laxatives, and stool softeners) have been approved to relieve the symptoms [9-11]. However, laxatives do not target the underlying pathophysiology, such as paradoxical anal contraction. Biofeedback therapy, an instrument-based learning process, can correct the incoordination of the abdominal, rectal, and anal sphincter pressures [12]. The efficacy of biofeedback therapy is reported to range from $44 \%$ to $100 \%$ in various clinical studies [13]. However, training requires complex processing and the training targets are fixed, meaning all patients receive the same training regardless of different anorectal motility and ability to achieve the training goal. A novel method of adaptive biofeedback $(\mathrm{ABF})$ training reportedly changes the training targets and protocols according to patients' anorectal motility. This method of ABF has shown to be superior to the traditional biofeedback training [14].

The frequency and duration of traditional biofeedback training are variable in different clinical trials [15-18]. On average, patients are asked to receive treatment for 3 months at a frequency of twice per week. The inconvenience and lengthy duration of biofeedback treatment often lead to poor compliance. We propose an intensive biofeedback therapy once a day or once every other day. The aim of the present study was to confirm the efficacy of $\mathrm{ABF}$ and compare the efficacy of intensive therapy with nonintensive therapy in patients with constipation-related symptoms.

\section{Materials and Methods}

A retrospective cohort study was conducted on subjects who had been treated with $\mathrm{ABF}$ for constipation-related symptoms between April 2012 and September 2013. The results were compared between the intensive therapy and nonintensive therapy in terms of constipation-related symptoms. The subjects were selected in this study according to the following inclusion/exclusion criteria.

2.1. Inclusion and Exclusion Criteria. The study enrolled men and women, aged $\geq 18$ years, with a history of constipation-related symptoms. Constipation-related symptoms are defined as follows: $<3$ bowel movements (BMs) per week on average, hard stools, low stool volume, sensation of incomplete evacuation, straining at defecation, or a need for manual maneuver to facilitate evacuation. Exclusion criteria included drug-induced constipation, metabolic, endocrine, neurological disorders, surgical obstruction, megacolon/megarectum, surgical obstruction, and pseudoobstruction. Other exclusion criteria were severe cardiovascular, renal, liver, or lung diseases.

\subsection{Outcomes and Data Collection}

2.2.1. Primary Outcomes. Patients rate the severity of constipation in terms of bowel movements with the three-point scale classification $[0=$ normal ( $\geq 3$ BMs per week), $1=$ mild (1-2 BMs per week), $2=$ severe $(<1 \mathrm{BMs}$ per week $)]$. Criteria for therapeutic effects are being cured (BMs changed from severe or mild to normal), being effective (BMs changed from severe to mild), and having no effect (BMs did not change).

2.2.2. Secondary Outcomes. Secondary outcome measures usage of medications, defecation difficulty, hard stools, straining, incomplete bowel movement, low stool volume, manual maneuver to facilitate, abdominal bloating, and anus discomfort. Symptoms of defecation difficulty, hard stools, incomplete bowel movement, low stool volume are described on a $0-3$ scale $(0=$ absent, $1=$ mild, $2=$ moderate, 3 = severe), manual maneuver to facilitate $[0=$ absent, $1=$ mild ( $<1$ time per week), $2=$ moderate (1-3 times per week), 3 = severe $(>3$ times per week $)$.

2.2.3. Impact on Social Activities and Work. The impact on social activities and work is rated on a $0-2$ scale where $0=$ absent, 1 = mild (a mild effect on normal social activities and normal work), and 2 = severe (a severe effect). Criteria for therapeutic effects are being cured (change from severe or mild to absent), being effective (change from severe to mild), and having no effect (no change).

2.3. Adaptive Biofeedback Training. Biofeedback training for the treatment of constipation is to train the relaxation of anal sphincter, enhance the sensory perception, and improve the rectoanal coordination. Training of rectoanal coordination is to increase the pushing effort as reflected by an increase in intra-abdominal/intrarectal pressures and synchronized relaxation reflected by a decrease in anal sphincter pressure. However, the traditional biofeedback training algorithm uses the fixed training target, it cannot increase (or decrease) the training strength or duration based on patient's capacity. On the other hand, the adaptive biofeedback training (ABT) (Ningbo Maida Medical Device Inc. Ningbo, China.) method uses the training strength and duration based on patient's own capacity and trains the patient at strength slightly above his or her own threshold with the purpose to gradually increase the strength threshold until the targeted threshold is met. It was reported to have a better efficacy for the treatment of constipation than the traditional biofeedback training method. Each patient received a total of 16 training sessions with each training session lasting half an hour.

Intensive Therapy. Patients were asked to receive intensive biofeedback therapy once a day or once every other day.

Nonintensive Therapy. Patients received nonintensive training twice a week in the motility lab.

2.4. Statistical Analysis. The data are expressed as mean \pm standard error. The paired-sample $t$-test was used to compare defecation difficulty, hard stools, straining, incomplete bowel movement, low stool volume, manual maneuver to facilitate, abdominal bloating, and anus discomfort before and after treatment with ABF. An independent $t$-test was used to compare the nonintensive therapy with the intensive therapy 


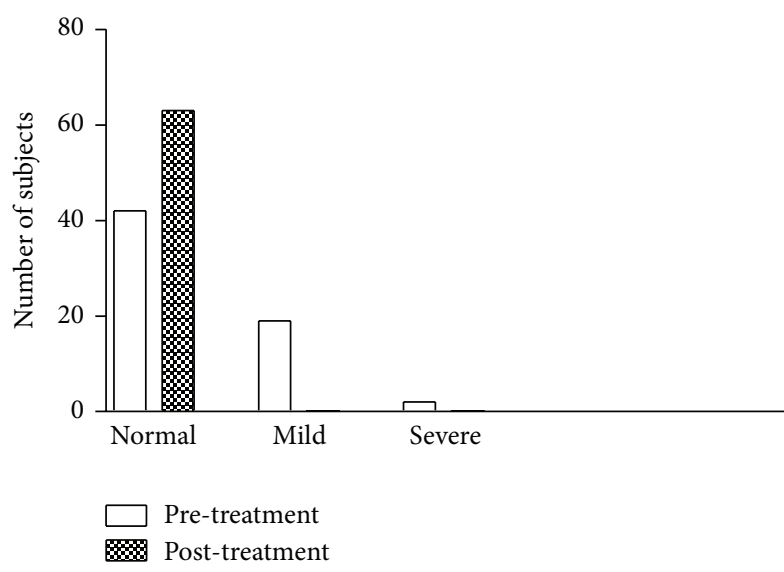

FIGURE 1: Effects of ABF on bowel movement (BM).

group. Data were considered statistically significant if $P<$ 0.05 .

\section{Result}

A total of 63 subjects met the inclusive criteria. 21 subjects had a long history of constipation defined as an average of $<3 \mathrm{BMs}$ per week. The mean age of the participants was $45.60 \pm 16.60$ and $42(66.66 \%)$ were women and 21 were men. There was no significant difference in age and gender between the two treatment groups.

After adaptive biofeedback training treatment, all constipation patients $(N=21)$ reported a significantly greater number of weekly bowel movements ( $\geq 3$ times) compared with the baseline ( $<3$ times). The cure rate of nonintensive therapy $(N=8)$ and intensive therapy $(N=13)$ both reached $100 \%$. None of the patients reported less than 3 BMs per week after the treatment (Figure 1). The usage of medications decreased considerably during the training period in both treatment groups compared to baseline. The medication usage at the start of treatment was $100 \%$ for nonintensive therapy group and $92.3 \%$ for intensive therapy group. During the treatment period, medication usage decreased to $12.5 \%$ for the nonintensive therapy group and $5.1 \%$ for the intensive therapy group (Figure 2).

As shown in Table 1, defecation difficulty, hard stools, and straining significantly improved with nonintensive therapy/intensive therapy compared with baseline $(P<0.05)$. Intensive therapy patients also reported significant improvements in incomplete BM. Intensive therapy also improved low stool volume $(P=0.006)$ and decreased manual maneuver frequency $(P=0.048)$. Both treatments significantly decreased abdominal bloating $(P<0.05)$. Nonintensive therapy, but not intensive therapy, significantly reduced the scores for anus discomfort ( 0 versus $0.48+0.87, P=$ 0.011 ; 0 versus $0.10+0.50, P=0.21$ ). However, there was no statistically difference between the two methods in all symptoms $(P>0.05)$.

Overall, $82.5 \%(N=52)$ of subjects reported that constipation symptoms interfered with normal social activities

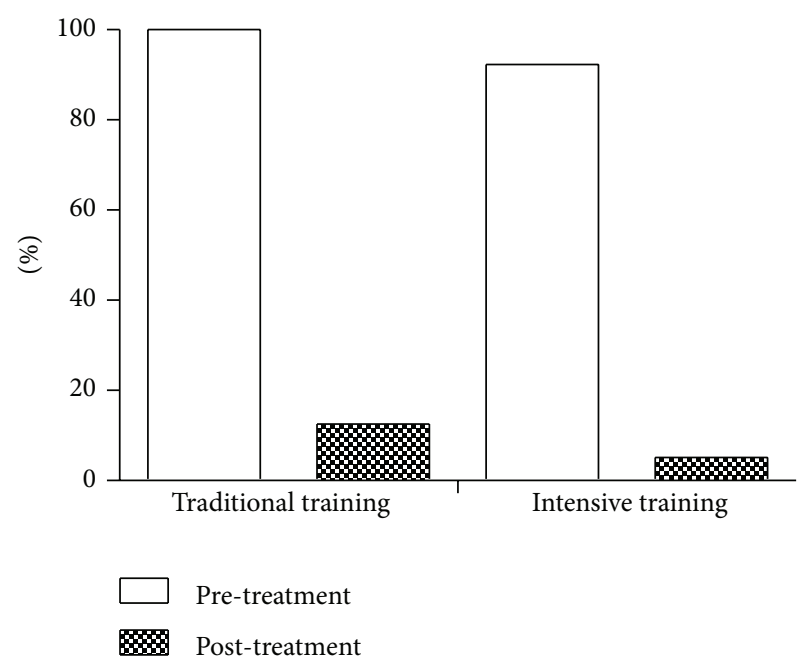

FIGURE 2: Usage of medications during the biofeedback training.

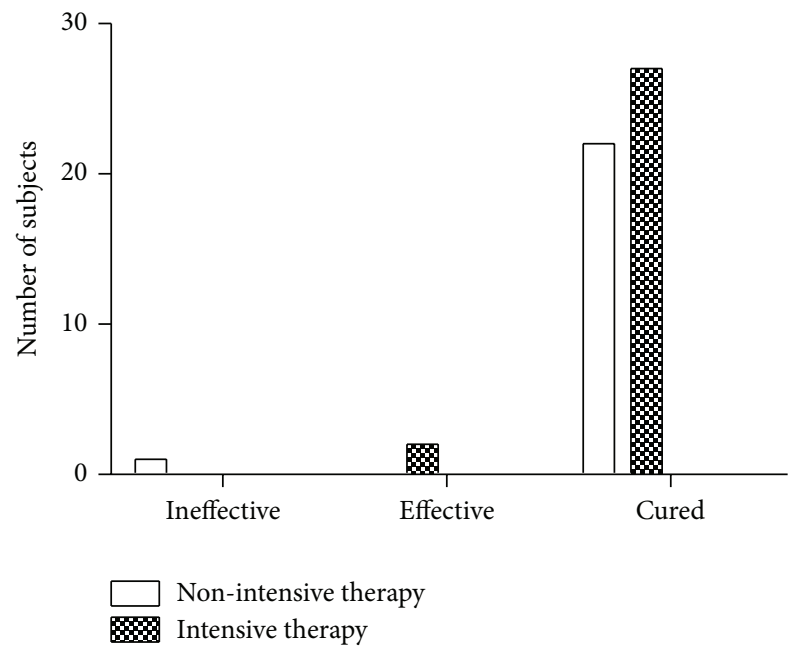

FIgURE 3: Improve the impact on social activities and work.

and normal work. The number of patients receiving either nonintensive therapy or intensive therapy who were cured was high (22 and 27, resp.). Only 1 patient with nonintensive therapy showed no improvement (Figure 3).

\section{Discussion}

The results of this study indicate that adaptive biofeedback training was effective in the treatment of patients with constipation-related symptoms. The adaptive biofeedback training was able to significantly increase weekly bowel movements. Patients also showed major improvement in defecation difficulty, hard stools and straining, incomplete $\mathrm{BM}$, low stool volume, manual maneuver to facilitate, and abdominal bloating. In the current study, adaptive biofeedback training also reduced the impact on social activities and work created by constipation-related symptoms. 
TABLE 1: Constipation-related symptoms before and after intensive therapy/nonintensive therapy.

\begin{tabular}{lcccc}
\hline & \multicolumn{2}{c}{ Intensive therapy } & \multicolumn{2}{c}{ Nonintensive therapy } \\
& Before training & After training & Before training & After training \\
\hline Defecation difficulty & $1.18+1.12$ & $0.13+0.41^{*}$ & $0.79+1.06$ & $0.17+0.48^{*}$ \\
Staining & $0.44+0.97$ & $0.05+0.22^{*}$ & $0.58+0.93$ & 0 \\
Incomplete BM & $0.41+0.82$ & $0.03+0.16^{*}$ & $0.25+0.68$ & $0.17+0.57$ \\
Low stool volume & $0.67+1.01$ & $0.26+0.50^{*}$ & $1.04+1.08$ & $0.04+0.20$ \\
Hard stools & $0.67+1.06$ & $0.10+0.31^{*}$ & $0.08+0.41$ & $0.13+0.45^{*}$ \\
Manual maneuver to facilitate & $0.23+0.71$ & $0^{*}$ & $0.96+1.20$ & $0.04+0.20^{*}$ \\
Abdominal bloating & $0.46+0.88$ & $0.03+0.16^{*}$ & $0.50+0.89$ & $0^{*}$ \\
Anus discomfort & $0.10+0.50$ & 0 &
\end{tabular}

Our results are consistent with the study conducted by $\mathrm{Xu}$ et al. [14] who recently reported that adaptive biofeedback training was more effective in improving bowel movements than those of conventional fixed biofeedback training $(3.4 \pm$ 1.3 versus $2.6 \pm 0.5, P<0.005)$. In this study, twenty-one constipation patients $(100 \%)$ had bowel movements of more than 3 times per week after ABF therapy. Chiarioni et al. [15] reported $82 \%$ of patients had $\geq 3$ bowel movements per week at 12-month follow-up after fixed biofeedback training. Only $29 \%$ patients reported $\geq 3$ bowel movements per week at 4 weeks of prucalopride therapy [19]. The ABF had a higher bowel movement response rate than fixed biofeedback training and laxative.

ABF significantly improved symptoms of constipation, such as defecation difficulty, incomplete BM, hard stools, and straining based on ROME III criteria [20]. Xu et al. [14] reported that $\mathrm{ABF}$ significantly improved these symptoms compared with fixed biofeedback training.

In addition, the impact of constipation symptoms on social activities and work was significantly decreased at the end of ABF. A growing evidence shows that constipation patients have a significantly impaired health-related quality of life compared with population norms [21-23]. Although this study did not use standard assessment tools to characterize quality of life, the results indicated that symptoms had an impact on social function. Other studies reported that fixed biofeedback training improved the quality of life scores compared with control group $[18,24]$.

In this study, we investigated the efficacy of intensive therapy compared to nonintensive therapy. In previous studies, patients were asked to receive nonintensive biofeedback training twice a week with a total of 4 to 6 sessions [25]. We proposed an intensive biofeedback therapy of which frequency was once a day or once every other day. There was no significant difference in constipation-related symptoms between the two treatment groups. Several randomized controlled trials had variable duration and number of biofeedback sessions, but the efficacy of therapy was similar $[15-18,26]$. But the intensive biofeedback therapy had short duration and may have better compliance.

In conclusion, treatment with adaptive biofeedback training produced significant improvement in bowel movements. $\mathrm{ABF}$ also significantly improved symptoms associated with constipation. The intensive biofeedback therapy did not seem to be superior to nonintensive therapy.

\section{Conflict of Interests}

The authors declare that there is no conflict of interests regarding the publication of this paper.

\section{Authors' Contribution}

Jing Tang and Zhihui Huang contributed equally to this paper.

\section{References}

[1] N. C. Suares and A. C. Ford, "Prevalence of, and risk factors for, chronic idiopathic constipation in the community: systematic review and meta-analysis," The American Journal of Gastroenterology, vol. 106, no. 9, pp. 1582-1591, 2011.

[2] E. Rey, A. Balboa, and F. Mearin, "Chronic constipation, irritable bowel syndrome with constipation and constipation with pain/discomfort: similarities and differences," The American Journal of Gastroenterology, vol. 109, no. 6, pp. 876-884, 2014.

[3] J. Belsey, S. Greenfield, D. Candy, and M. Geraint, "Systematic review: impact of constipation on quality of life in adults and children," Alimentary Pharmacology and Therapeutics, vol. 31, no. 9, pp. 938-949, 2010.

[4] C. Dennison, M. Prasad, A. Lloyd, S. K. Bhattacharyya, R. Dhawan, and K. Coyne, "The health-related quality of life and economic burden of constipation," PharmacoEconomics, vol. 23, no. 5, pp. 461-476, 2005.

[5] W. Ashraf, F. Park, J. Lof, and E. M. M. Quigley, "An examination of the reliability of reported stool frequency in the diagnosis of idiopathic constipation," The American Journal of Gastroenterology, vol. 91, no. 1, pp. 26-32, 1996.

[6] A. Lembo and M. Camilleri, "Chronic constipation," The New England Journal of Medicine, vol. 349, no. 14, pp. 1360-1368, 2003.

[7] S. Gonlachanvit and T. Patcharatrakul, "Causes of idiopathic constipation in Thai patients: associations between the causes and constipation symptoms as defined in the Rome II criteria," Journal of the Medical Association of Thailand, vol. 87, supplement 2, pp. S22-S28, 2004.

[8] S. Shahid, Z. Ramzan, A. H. Maurer, H. P. Parkman, and R. S. Fisher, "Chronic idiopathic constipation: More than a simple 
colonic transit disorder," Journal of Clinical Gastroenterology, vol. 46, no. 2, pp. 150-154, 2012.

[9] L. W. Liu, "Chronic constipation: current treatment options," Canadian Journal of Gastroenterology, vol. 25, pp. 22B-28B, 2011.

[10] E. Klaschik, F. Nauck, and C. Ostgathe, "Constipation: modern laxative therapy," Supportive Care in Cancer, vol. 11, no. 11, pp. 679-685, 2003.

[11] M. El-Salhy, R. Svensen, J. G. Hatlebakk, O. H. Gilja, and T. Hausken, "Chronic constipation and treatment options (Review)," Molecular Medicine Reports, vol. 9, no. 1, pp. 3-8, 2014.

[12] S. S. Rao, "Biofeedback therapy for constipation in adults," Best Practice and Research: Clinical Gastroenterology, vol. 25, no. 1, pp. 159-166, 2011.

[13] S. Heymen, K. R. Jones, Y. Scarlett, and W. E. Whitehead, "Biofeedback treatment of constipation: a critical review," Diseases of the Colon \& Rectum, vol. 46, no. 9, pp. 1208-1217, 2003.

[14] Y. Xu, X. Li, F. Xu, D. W. Lu, J. Chen, and J. D. Z. Chen, "A novel method of adaptive biofeedback training for dyssynergic defecation," Neurogastroenterology \& Motility, vol. 25, supplement 1, pp. 13-45, 2013.

[15] G. Chiarioni, L. Salandini, and W. E. Whitehead, "Biofeedback benefits only patients with outlet dysfunction, not patients with isolated slow transit constipation," Gastroenterology, vol. 129, no. 1, pp. 86-97, 2005.

[16] S. S. C. Rao, K. Seaton, M. Miller et al., "Randomized controlled trial of biofeedback, sham feedback, and standard therapy for dyssynergic defecation," Clinical Gastroenterology and Hepatology, vol. 5, no. 3, pp. 331-338, 2007.

[17] S. S. C. Rao, J. Valestin, C. K. Brown, B. Zimmerman, and K. Schulze, "Long-term efficacy of biofeedback therapy for dyssynergic defecation: randomized controlled trial," The American Journal of Gastroenterology, vol. 105, no. 4, pp. 890-896, 2010.

[18] S. Heymen, Y. Scarlett, K. Jones, Y. Ringel, D. Drossman, and W. E. Whitehead, "Randomized, controlled trial shows biofeedback to be superior to alternative treatments for patients with pelvic floor dyssynergia-type constipation," Diseases of the Colon and Rectum, vol. 50, no. 4, pp. 428-441, 2007.

[19] E. M. M. Quigley, L. Vandeplassche, R. Kerstens, and J. Ausma, "Clinical trial: the efficacy, impact on quality of life, and safety and tolerability of prucalopride in severe chronic constipation-a 12-week, randomized, double-blind, placebocontrolled study," Alimentary Pharmacology and Therapeutics, vol. 29, no. 3, pp. 315-328, 2009.

[20] D. A. Drossman and D. L. Dumitrascu, "Rome III: new standard for functional gastrointestinal disorders," Journal of Gastrointestinal and Liver Diseases, vol. 15, no. 3, pp. 237-241, 2006.

[21] A. Wald, C. Scarpignato, M. A. Kamm et al., "The burden of constipation on quality of life: results of a multinational survey," Alimentary Pharmacology and Therapeutics, vol. 26, no. 2, pp. 227-236, 2007.

[22] A. K. Tuteja, N. J. Talley, S. K. Joos, J. V. Woehl, and D. H. Hickam, "Is constipation associated with decreased physical activity in normally active subjects?" The American Journal of Gastroenterology, vol. 100, no. 1, pp. 124-129, 2005.

[23] S. S. Rao, K. Seaton, M. J. Miller et al., "Psychological profiles and quality of life differ between patients with dyssynergia and those with slow transit constipation," Journal of Psychosomatic Research, vol. 63, no. 4, pp. 441-449, 2007.
[24] S. L. Hart, J. W. Lee, J. Berian, T. R. Patterson, A. del Rosario, and M. G. Varma, "A randomized controlled trial of anorectal biofeedback for constipation," International Journal of Colorectal Disease, vol. 27, no. 4, pp. 459-466, 2012.

[25] E. Battaglia, A. M. Serra, G. Buonafede et al., "Long-term study on the effects of visual biofeedback and muscle training as a therapeutic modality in pelvic floor dyssynergia and slowtransit constipation," Diseases of the Colon and Rectum, vol. 47, no. 1, pp. 90-95, 2004.

[26] G. Chiarioni, W. E. Whitehead, V. Pezza, A. Morelli, and G. Bassotti, "Biofeedback is superior to laxatives for normal transit constipation due to pelvic floor dyssynergia," Gastroenterology, vol. 130, no. 3, pp. 657-664, 2006. 


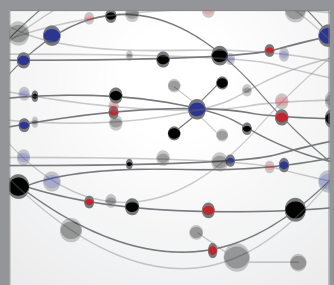

The Scientific World Journal
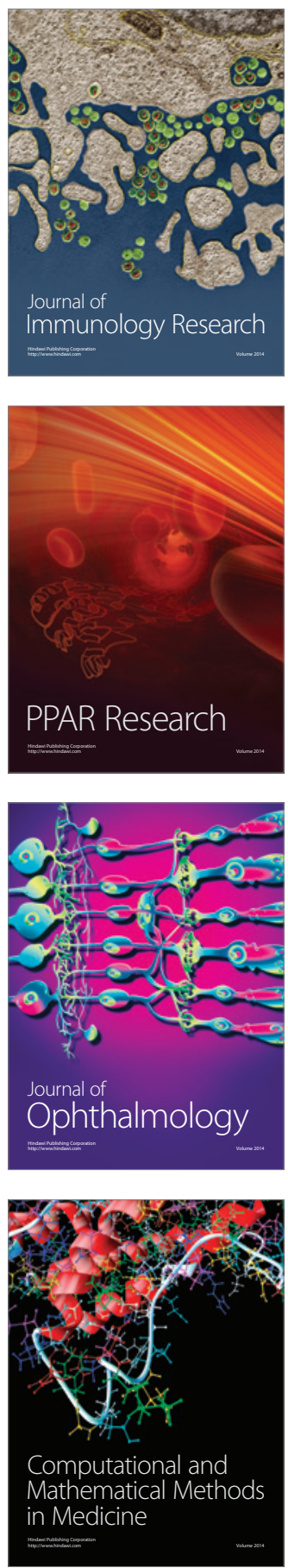

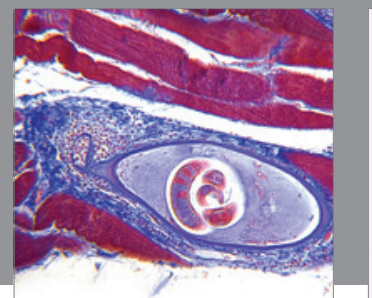

Gastroenterology

Research and Practice
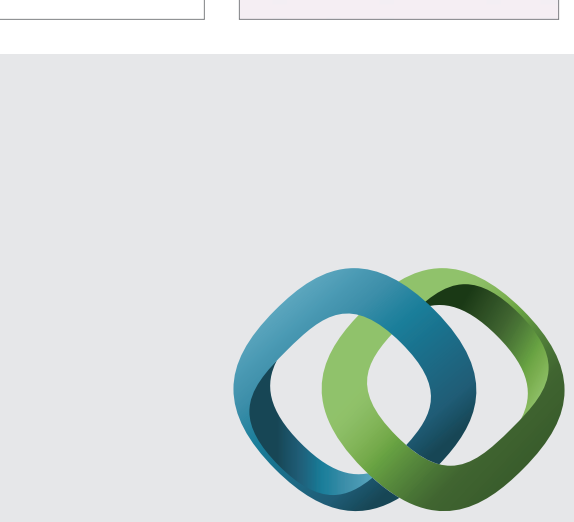

\section{Hindawi}

Submit your manuscripts at

http://www.hindawi.com
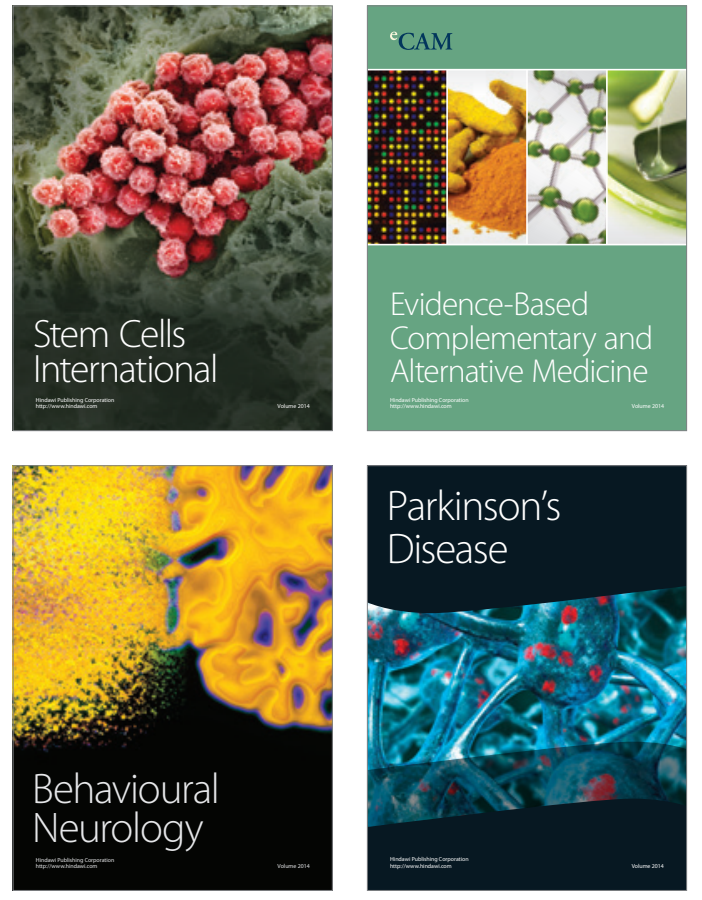
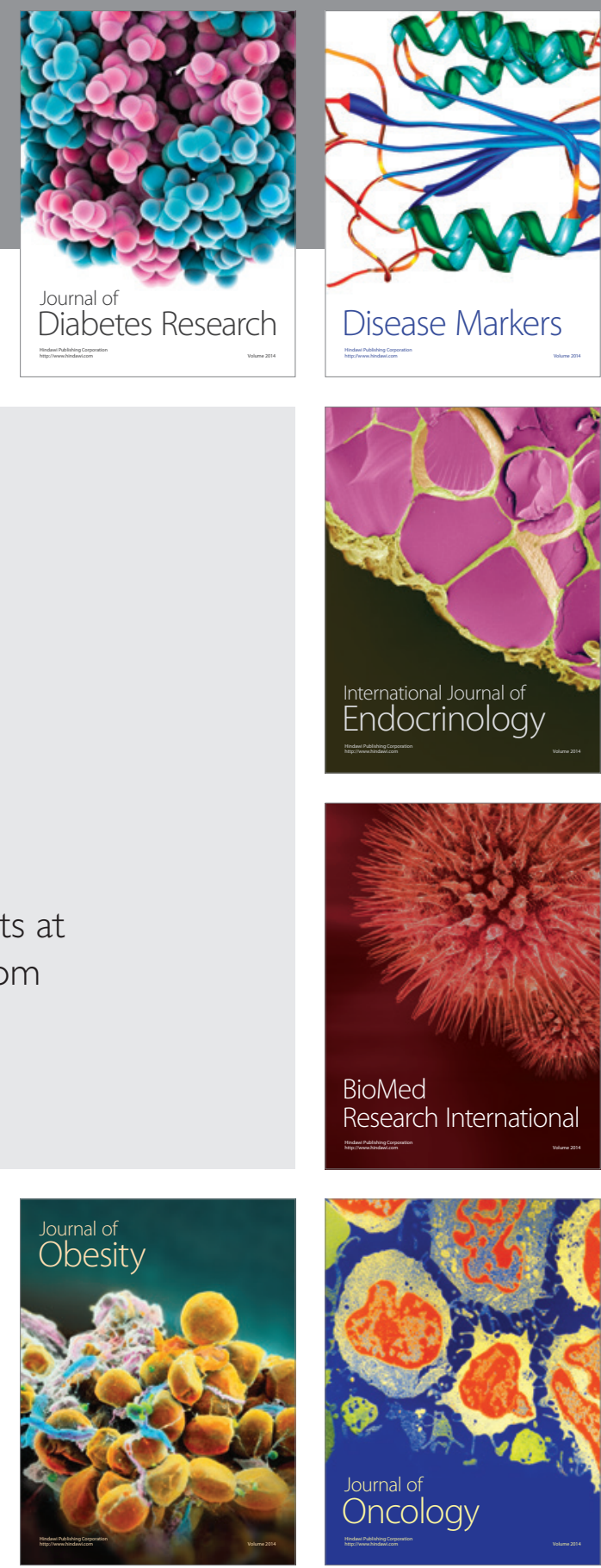

Disease Markers
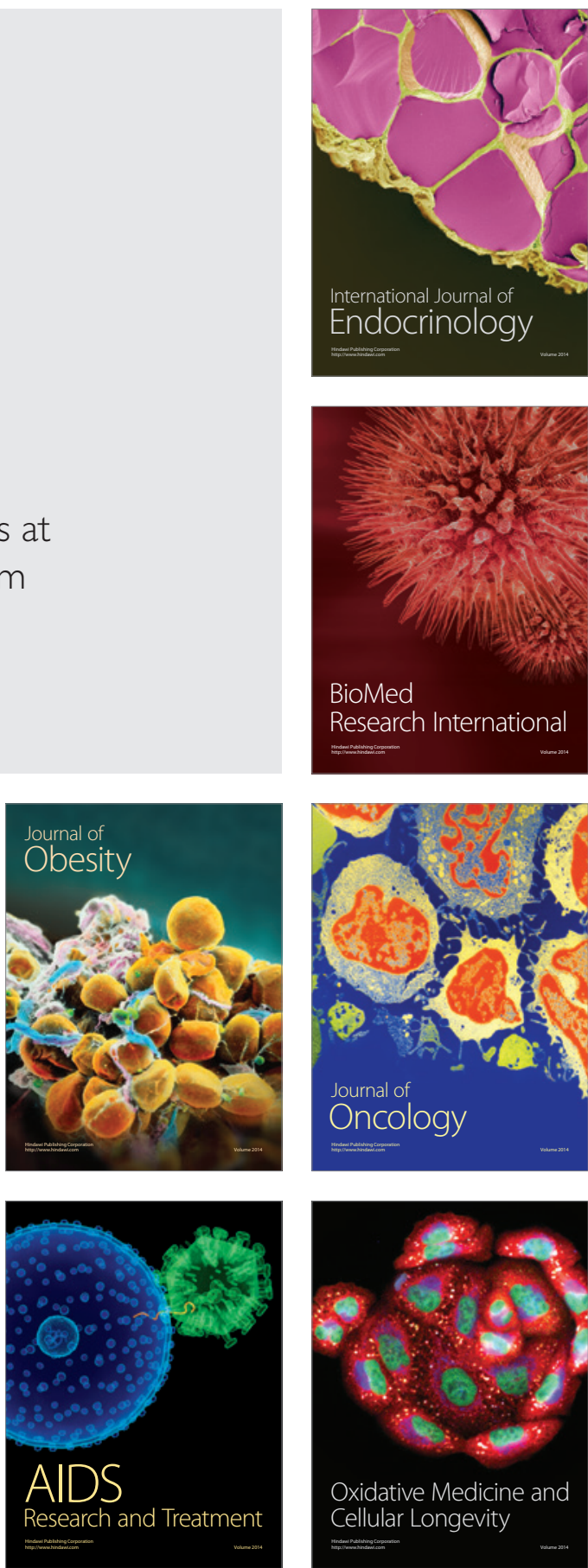